\title{
Efficacy of continuous local cryotherapy following total hip arthroplasty
}

\author{
Kentaro Iwakiri ${ }^{1, *}$, Akio Kobayashi ${ }^{1}$, Yuichi Takeuchi ${ }^{1}$, Yusuke Kimura ${ }^{1}$, Yoichi Ohta ${ }^{2}$, \\ and Hiroaki Nakamura ${ }^{2}$ \\ ${ }^{1}$ Department of Orthopaedic Surgery, Shiraniwa Hospital, 6-10-1 Shiraniwadai Ikoma-city, 630-0136 Nara, Japan \\ 2 Department of Orthopaedic Surgery, Osaka City University Graduate School of Medicine, 1-4-3 Asahi-machi Abeno-ku Osaka-city, \\ 545-8585 Osaka, Japan
}

Received 26 February 2019, Accepted 7 April 2019, Published online 3 May 2019

\begin{abstract}
Background: Cryotherapy is rarely reported on the usefulness of cryotherapy applied after total hip arthroplasty (THA), and there are no reports about patient satisfaction against the cryotherapy following THA. The aim of this study was whether cryotherapy can be useful for relieving pain, reducing blood loss, and swelling, and improving patient satisfaction after THA.

Methods: Thirty patients who had undergone THA were treated by a controlled cooling device for $72 \mathrm{~h}$ following THA (defined as the cryotherapy group). The other 30 patients without cryotherapy were not treated with cryotherapy (defined as the control group). Blood samples (creatine kinase, and C-reactive protein), estimated blood loss, visual analog scale (VAS) of pain score, total doses of diclofenac sodium suppository used for pain relief, thigh swelling, Western Ontario and McMaster Universities Osteoarthritis Index (WOMAC) score, and adverse outcomes were compared between two groups.

Results: Thigh circumference, measured on only day 4 postoperatively, was significantly lower in the cryotherapy than in the control group. Furthermore, patient satisfaction on postoperative days 4 and 7 was significantly higher in the cryotherapy than in the control group. There were no significant differences in other outcomes between groups.

Conclusions: These results support the potential benefit of cryotherapy for the reduction of swelling, and patient satisfaction during postoperative recovery of patients undergoing THA, even in the presence of periarticular injection and tranexamic acid administration for the prevention of postoperative pain and bleeding. Postoperative cryotherapy is a potentially simple, noninvasive, and relatively inexpensive option for post-THA management.
\end{abstract}

Key words: Cryotherapy, Total hip arthroplasty, Swelling, VAS score, Patient satisfaction.

\section{Introduction}

Total hip arthroplasty (THA) has become one of the mainstays for surgical management of end-stage arthritis of the hip [1]. In THA, cryotherapy has occasionally been reported to be useful for pain relief [2-5]. Regarding the mechanism underlying the effects of cryotherapy, the soft tissue is cooled to lower the intra-articular temperature, thereby reducing both the induction of neural signals and local blood flow [6]. Consequently, inflammatory responses are prevented. Thus, cryotherapy is considered to be useful for reducing blood loss and relieving local pain and swelling [7]. Moreover, cryotherapy has the advantages of being both safe and inexpensive. However, the majority of reports on cryotherapy describe the postoperative course after total knee arthroplasty (TKA) [8-10],

\footnotetext{
*Corresponding author: kenpiecekenpiece@yahoo.co.jp
}

with few reports on the usefulness of cryotherapy applied after THA [2-5]. Our present report describes the first survey to focus on postoperative swelling and patient satisfaction after THA.

This study aimed to examine whether continuous local cryotherapy is useful for relieving pain, and reducing blood loss, swelling, and the duration of hospitalization, as well as for improving patient satisfaction and postoperative outcomes after THA. We hypothesized that cryotherapy would reduce postoperative pain and improve patient satisfaction, thereby leading to more rapid progress with ambulatory rehabilitation.

\section{Materials and methods}

We conducted a single-center retrospective study. This study protocol was approved by the institutional review board. 
Table 1. Demographic data of patients in two groups.

\begin{tabular}{lccc}
\hline & Control & Cryotherapy & $p$ value \\
\hline$n$ & 30 & 30 & - \\
Female/male & $29 / 1$ & $29 / 1$ & $0.1+9.6$ \\
Age (years) & $67.6 \pm 8.9$ & $24.1 \pm 3.1$ & $0.74^{*}$ \\
BMI (kg/m ${ }^{2}$ ) & $23.9 \pm 3.1$ & $29 / 1$ & - \\
OA/ION & $29 / 1$ & $451.1 \pm 240.6$ & $0.274^{*}$ \\
Blood loss during surgery $(\mathrm{mL})$ & $391.2 \pm 173.5$ & $100.4 \pm 16.7$ & $0.367^{*}$ \\
Duration of surgery (min.) & $96.6 \pm 15.6$ & $25.7 \pm 5.6$ & $0.558^{*}$ \\
Hospital stay (day) & $24.7 \pm 7.0$ & & \\
\hline
\end{tabular}

Data are shown as mean \pm standard deviation or numbers.

OA, osteoarthritis; ION, idiopathic osteonecrosis of the femoral head.

* Mann-Whitney $U$ test.

All procedures performed in studies involving human participants were in accordance with the ethical standards of the institutional and/or national research committee and with the 1964 Helsinki declaration and its later amendments or comparable ethical standards.

\section{Study population}

We reviewed 67 patients who had undergone primary THA for osteoarthritis or osteonecrosis of the hip at our institution between November 2012 and February 2015. We excluded patients who had simultaneous or staged bilateral THA or for revision THA. The subjects were stratified, according to the duration of the surgery from March to October in 2013 with our study group consisting of 30 patients (following their approval for participation) who had received cryotherapy and from November 2012 to February 2013 and after November 2013 with the control group consisting of 30 patients who did not receive cryotherapy. Seven patients were excluded without the approval for participation of the study. Demographic data are listed in Table 1.

\section{Surgery}

All patients underwent cementless THA via an anterolateral approach in the lateral position under general anesthesia. All cases were performed by a single surgeon. All patients routinely administered an intraoperative periarticular injection that we have previously reported (40-mL cocktail including ropivacaine, epinephrine, ketoprofen, methylprednisolone sodium, and normal saline solution) [11]. As prevention for perioperative bleeding, $2 \mathrm{~g}$ of tranexamic acid was injected (1 g intravenously before surgery and $1 \mathrm{~g}$ under the tensor fascia tissue after suture of the tensor fascia tissue). None of the patients had a drainage catheter placed. Wound closure strips (LEUKOSTRIP, Smith and Nephew, USA) were applied to the suture wound and covered with a transparent dressing of absorbent foam (OPSITE Post-Op Visible, Smith and Nephew, USA).

\section{Interventions}

Postoperatively in the cryotherapy group, a cooling pad $(28 \times 29 \mathrm{~cm})$ wrapped in a waterproof cover was applied to the dressing immediately after surgery (Figure 1a), and the surgical wound and the thigh were fixed with a commercially available cloth anchor bandage (Sigmax, Tokyo, Japan). A controlled cooling device (Icing System CF3000, Sigmax, Tokyo, Japan) (Figure 1b) was initiated immediately after surgery and operated constantly for $72 \mathrm{~h}$ at the cooling temperature of $5{ }^{\circ} \mathrm{C}$. The subjects were instructed to wear the cooling device whenever on bed. The same procedure was treated for the control group, involving the fixation with the cloth anchor bandage for $72 \mathrm{~h}$ without the cooling procedure. The intermittent compression device for thrombosis prophylaxis was used for $24 \mathrm{~h}$ immediately after surgery.

\section{Perioperative medications}

For all subjects, a nonsteroidal anti-inflammatory drug (200 mg of celecoxib) was administered twice a day for the first 7 days after surgery. The diclofenac sodium suppository ( $25 \mathrm{mg}$ ) was utilized for the rescue analgesia. The cephalosporin was intravenously administered perioperatively as an antibiotic prophylaxis and every $6 \mathrm{~h}$ for $24 \mathrm{~h}$ after surgery. No thromboprophylaxis was treated. Every patient received an autologous blood transfusion $(400 \mathrm{~mL})$ on postoperative day 1 .

\section{Outcome measurements}

\section{Primary outcomes}

Primary outcomes included laboratory data (creatine kinase $[\mathrm{CK}]$, and C-reactive protein [CRP] obtained preoperatively and on postoperative days $1,4,7,14$, and 21), estimated blood loss [12], visual analog pain score on motion obtained once a day during physical therapy (VAS on motion obtained preoperatively and on postoperative days $4,7,14$, and 28), and thigh swelling (thigh circumference measured at $5 \mathrm{~cm}$ proximal to the patella superior border obtained preoperatively and on postoperative days $4,7,14$, and 28). The measurements of VAS score and thigh circumference were calculated by the physical therapist in the blinded fashion.

The estimated blood loss was calculated by the difference between the preoperative hemoglobin and the lowest postoperative level. The estimated blood loss was measured by the Nadler formula [12] at postoperative day 4 after surgery. 


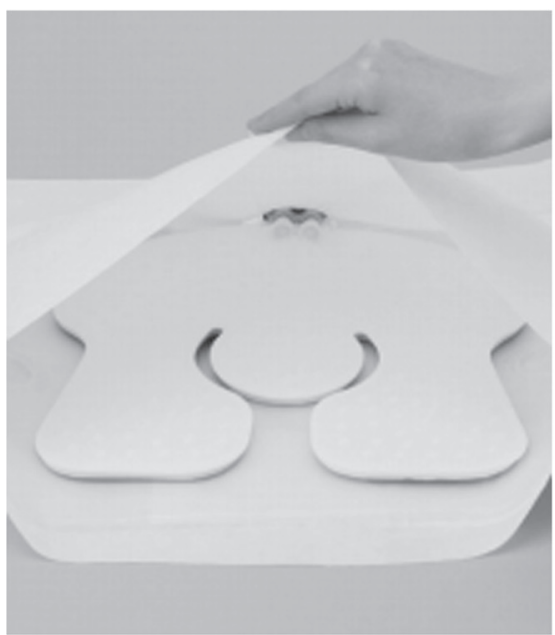

(a)

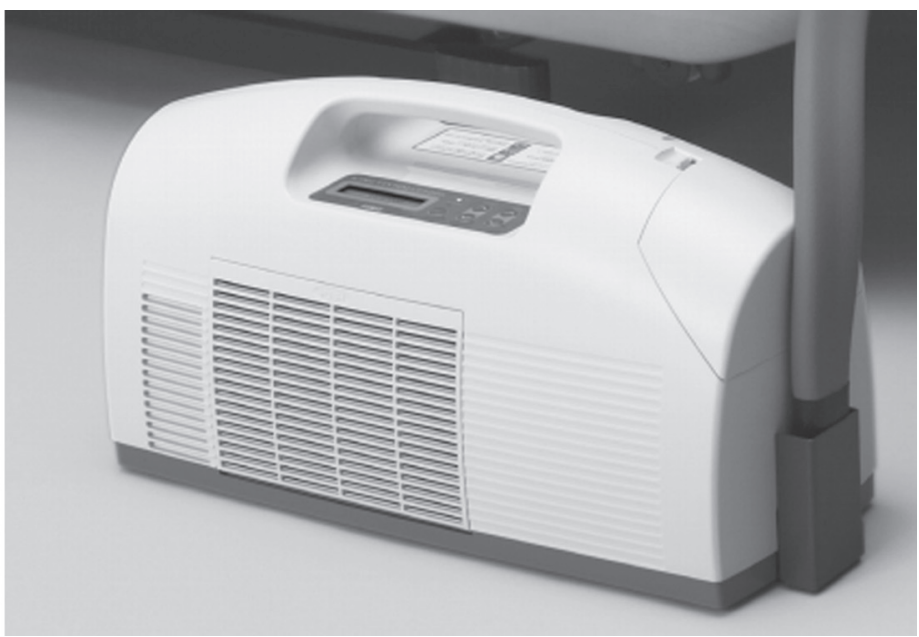

(b)

Figure 1. (a) A cooling pad $(28 \times 29 \mathrm{~cm})$ wrapped in a waterproof cover for the surgical wound and the thigh (Sigmax, Tokyo, Japan). (b) A computer-controlled cooling device (Icing System CF3000, Sigmax, Tokyo, Japan).

The VAS score was defined from $0 \mathrm{~mm}$ (indicating no pain) to $100 \mathrm{~mm}$ (indicating extreme pain) in 10-mm increments. It was also recorded if a diclofenac sodium suppository was used as rescue analgesia within 21 days postoperatively. The ratio of postoperative thigh circumference to preoperative thigh circumference was measured to compare thigh swelling between the two groups.

\section{Secondary outcomes}

Secondary outcomes measured were the WOMAC Index (obtained preoperatively, and 3 months postoperatively), patient satisfaction of walking ability (VAS score obtained on postoperative days 4, 7, 14, and 28) and adverse events during this study, including surgical site infection, peroneal nerve palsy, wound complications, and deep venous thrombosis (DVT). The WOMAC Index score ranged from 100 points (indicating poor assessment) to 0 points (indicating excellent assessment for pain, stiffness, and function). The VAS score of patient satisfaction of walking ability ranged from $0 \mathrm{~mm}$ (indicating poor satisfaction) to $100 \mathrm{~mm}$ (indicating excellent satisfaction) in 10$\mathrm{mm}$ increments [1].

\section{Statistical analysis}

The Mann-Whitney $U$-test for continuous variables and Fisher's exact probability test for categorical variables were used to analyze the primary and secondary outcomes statistically between the two groups. The $p$ value of less than 0.05 was considered to indicate a statistically significant difference. All statistical analyses were done using the SPSS statistics version 22.0 (IBM Corp., USA).

\section{Results}

Thirty patients in the cryotherapy group and thirty patients in the control group were included and compared in the study
(Table 1). The average age of all subjects at surgery was $67.9 \pm 9.2$ years. There were 58 women and 2 men. The preoperative diagnosis was osteoarthritis of the hip in 58 patients and idiopathic osteonecrosis of the hip in two patients. There were no significant differences in age $(p=0.824)$, BMI $(p=0.787)$, blood loss during surgery ( $p=0.274)$, the duration of surgery ( $p=0.367)$, or duration of hospitalization $(p=0.558)$ between the two groups (Table 1).

\section{Primary outcomes}

There were no significant differences between the two groups in CK levels, CRP levels, estimated blood loss, or pain VAS scores. However, the total amount of diclofenac sodium suppository tended to be significantly lower in the cryotherapy group ( $p=0.070)$. Thigh circumference, measured postoperatively only on day 4 , was significantly lower in the cryotherapy group than in the control group ( $p=0.045$ ) (Table 2). The overall VAS reduction was higher in the control group than in the cryotherapy group, but there were no significant differences between the two groups $(p=0.52)$.

\section{Secondary outcomes}

There were no significant differences in WOMAC scores between the two groups. However, patient satisfaction of walking ability measured on postoperative days 4 and 7 was significantly higher in the cryotherapy group than in the control group ( $p=0.03$ and 0.04 , respectively). No complications such as skin problems, infection, symptomatic DVT, or neuroparalysis were observed in either group (Table 3 ).

\section{Discussion}

In recent years, cryotherapy after TKA, shoulder arthroscopic surgery, and carpal tunnel release has sporadically been 
Table 2. Primary outcomes.

\begin{tabular}{|c|c|c|c|}
\hline & Control & Cryotherapy & $p$ value \\
\hline \multicolumn{4}{|l|}{ Laboratory data } \\
\hline \multicolumn{4}{|l|}{ CK (IU/L) } \\
\hline Pre-op. & $96.6 \pm 63.7$ & $107.0 \pm 79.4$ & 0.578 \\
\hline Day 1 & $439.1 \pm 201.4$ & $467.1 \pm 234.8$ & 0.622 \\
\hline Day 4 & $447.1 \pm 233.6$ & $525.7 \pm 266.8$ & 0.230 \\
\hline Day 7 & $183.2 \pm 106.8$ & $181.9 \pm 144.5$ & 0.054 \\
\hline Day 14 & $53.3 \pm 13.6$ & $67.5 \pm 38.9$ & 0.067 \\
\hline Day 21 & $57.3 \pm 15.4$ & $60.5 \pm 31.7$ & 0.668 \\
\hline \multicolumn{4}{|l|}{ CRP (mg/dL) } \\
\hline Pre-op. & $0.21 \pm 0.24$ & $0.13 \pm 0.15$ & 0.145 \\
\hline Day 1 & $1.93 \pm 0.93$ & $1.50 \pm 0.69$ & 0.056 \\
\hline Day 4 & $4.59 \pm 3.05$ & $4.57 \pm 2.74$ & 0.971 \\
\hline Day 7 & $1.27 \pm 1.06$ & $1.42 \pm 1.02$ & 0.574 \\
\hline Day 14 & $0.38 \pm 0.37$ & $0.44 \pm 0.68$ & 0.701 \\
\hline Day 21 & $0.28 \pm 0.27$ & $0.32 \pm 0.48$ & 0.719 \\
\hline Estimated blood loss (mL) & $784.7 \pm 293.3$ & $699.6 \pm 296.2$ & 0.268 \\
\hline \multicolumn{4}{|l|}{ Pain VAS } \\
\hline Pre-op. & $29.3 \pm 33.9$ & $24.1 \pm 28.5$ & 0.536 \\
\hline Day 4 & $9.3 \pm 13.6$ & $11.5 \pm 19.3$ & 0.624 \\
\hline Day 7 & $7.1 \pm 9.7$ & $11.5 \pm 17.3$ & 0.243 \\
\hline Day 14 & $5.4 \pm 9.4$ & $9.4 \pm 17.8$ & 0.294 \\
\hline Day 28 & $1.2 \pm 2.2$ & $3.3 \pm 7.1$ & 0.342 \\
\hline \multicolumn{4}{|l|}{$\begin{array}{l}\text { Thigh girth } \\
\text { (the ratio of Post/Pre) }\end{array}$} \\
\hline Pre-op. & 1 & 1 & \\
\hline Day 4 & $1.067 \pm 0.055$ & $1.041 \pm 0.038$ & 0.045 \\
\hline Day 7 & $1.047 \pm 0.057$ & $1.062 \pm 0.045$ & 0.267 \\
\hline Day 14 & $1.006 \pm 0.030$ & $1.021 \pm 0.048$ & 0.156 \\
\hline Day 28 & $1.007 \pm 0.026$ & $0.946 \pm 0.270$ & 0.246 \\
\hline \multicolumn{4}{|c|}{$\begin{array}{l}\text { Total amount of dicrofenac } \\
\text { sodium suppository }(\mathrm{mg})\end{array}$} \\
\hline & $72.2 \pm 79.5$ & $31.7 \pm 60.9$ & 0.070 \\
\hline
\end{tabular}

Data are shown as mean \pm standard deviation.

Table 3. Secondary outcomes.

\begin{tabular}{lccc}
\hline & Control & Cryotherapy & $p$-Value \\
\hline WOMAC & & & \\
$\quad$ Pre-op & $37.0 \pm 17.0$ & $38.9 \pm 20.5$ & 0.698 \\
$\quad$ Post-op 3 months & $15.0 \pm 11.8$ & $13.9 \pm 10.6$ & 0.408 \\
Patient-satisfaction & & & \\
$\quad$ of walking ability & $38 \pm 25$ & $62 \pm 16$ & 0.030 \\
Day 4 & $48 \pm 33$ & $67 \pm 17$ & 0.040 \\
Day 7 & $51 \pm 31$ & $67 \pm 20$ & 0.080 \\
Day 14 & $61 \pm 31$ & $75 \pm 20$ & 0.120 \\
Day 28 & & & \\
Adverse events & 0 & 0 & - \\
$\quad$ Wound complications & 0 & 0 & - \\
Surgical site infections & 0 & 0 & - \\
$\quad$ Nerve palsy & 0 & 0 & - \\
$\quad$ Deep venous thrombosis & & & \\
\hline
\end{tabular}

Data are shown as mean \pm standard deviation or numbers.

reported to be useful for postoperative pain relief and prevention of swelling [8-10]. However, only a few reports have described the effects of continuous cryotherapy applied to the hip joints, which are located deeper than the knee and hand joints, following THA [2-5].
Saito et al. reported the use of continuous cryotherapy after THA to be effective for pain relief (according to VAS score) only in the early postoperative period, while no useful effects were noted for hematological data (i.e., blood loss, CK, and CRP) [4]. Moreover, Leegwater et al. reported that a combination of cryotherapy and compression slightly reduced bleeding in the early postoperative period [2]. In the present study, we, for the first time, have added thigh swelling and patient satisfaction of walking ability assessments in order to clarify the effects of cryotherapy. Contrary to previous reports, there were no significant differences in pain VAS scores and estimated blood loss between the control and cryotherapy groups. Otherwise, on postoperative day 4, swelling was significantly suppressed, based on thigh circumference, and the total amount of diclofenac sodium suppository given tended to be reduced in the cryotherapy group. Because a periarticular multimodal injection was used in all patients for postoperative pain relief at our institution, the VAS scores might have been kept low enough to mask any difference between the two groups.

The mechanisms by which cryotherapy relieves pain and prevents swelling are considered to involve the constriction of blood vessels to reduce blood flow and edema [6]. Moreover, cryotherapy prevents inflammation by lowering tissue metabolic rates and inhibiting enzymatic activity that was representative in prostaglandin $E_{2}$ as an indicator of pain and inflammation [13]. Consequently, secondary tissue damage is prevented. The prevention of postoperative swelling of the lower limbs reduces the incidence of thrombogenesis in the early postoperative period [14]. At our institution, tranexamic acid was injected intravenously before surgery and beneath the tensor fascia tissue after suture of the tensor fascia tissue to all patients for the prevention of postoperative bleeding, and none of the patients had a drainage catheter placed for the improvement of patient satisfaction. In the present study, significant prevention of swelling, as evidenced by reduced thigh circumference on postoperative day 4 , can presumably be attributed to the use of cryotherapy. As bleeding and swelling were mild in all patients because of the effect of the tranexamic acid injection and periarticular cocktail injection, the effects of cryotherapy might have been less evident $[15,16]$.

There were a few limitations in this study. First, the patients in the cryotherapy group were instructed to wear the cooling device whenever on bed, but there was variation in the duration of cooling time over the 72-hour period. This factor was not considered in this study. Secondly, this was a retrospective study. Third, the thigh circumference at $5 \mathrm{~cm}$ proximal to the patella superior border was measured. It is unknown how much the thigh circumference at $5 \mathrm{~cm}$ proximal to the patella superior border reflects thigh swelling. Fourth, there is no evidence whether the cooling temperature of $5{ }^{\circ} \mathrm{C}$ is suitable for the cryotherapy. Last, there might be potentially some bias in the improved satisfaction scores for patients who were treated with cryotherapy. Any other intervention except for cryotherapy might have been associated with improved satisfaction.

The present results revealed that continuous cryotherapy is useful for reducing swelling, and improving patient satisfaction of walking ability, even when a peri-articular multimodal cocktail is injected for postoperative pain relief or tranexamic acid is injected for the prevention of postoperative bleeding. 
Thus, cryotherapy may allow early postoperative rehabilitation and consequently reduce the risk of postoperative DVT.

Postoperative cryotherapy is a potentially simple, noninvasive, and relatively inexpensive option for post-THA management.

\section{Conclusion}

This study examined whether continuous cryotherapy can be useful for relieving pain, and reducing blood loss, swelling, patient satisfaction, and the duration of hospitalization after THA. Continuous cryotherapy was effective in the immediate postoperative period for reducing swelling and minimizing the use of diclofenac sodium suppository, and improving patient satisfaction of walking ability, even when a periarticular multimodal cocktail was injected for postoperative pain relief or tranexamic acid was injected for prevention of postoperative bleeding.

\section{Conflict of interest}

I certify that each of us has no financial conflict of interest (e.g., consultancies, stock ownership, equity interest, patent/ licensing arrangements, etc) in connection with this article.

Acknowledgements. We thank Yusuke Hori and Koji Mandai in Osaka City University Graduate School of Medicine for the assistants of the orthopedic surgery, and Yusuke Kimura in the department of rehabilitation at Shiraniwa hospital. We did not receive grants or outside funding in support of their research or preparation of this manuscript.

\section{References}

1. Espehaug B, Havelin LI, Engesaeter LB, Langeland N, Vollset SE (1998) Patient satisfaction and function after primary and revision total hip replacement. Clin Orthop Relat Res 351, 135-148.

2. Leegwater NC, Willems JH, Brohet R, Nolte PA (2012) Cryocompression therapy after elective arthroplasty of the hip. Hip Int 22(5), 527-533.
3. Ni SH, Jiang WT, Guo L, Jin YH, Jiang TL, Zhao Y (2015) Cryotherapy on postoperative rehabilitation of joint arthroplasty. Knee Surg Sports Traumatol Arthrosc 23(11), 3354-3361.

4. Saito N, Horiuchi H, Kobayashi S, Nawata M, Takaoka K (2004) Continuous local cooling for pain relief following total hip arthroplasty. J Arthroplasty 19(3), 334-337.

5. Scarcella JB, Cohn BT (1995) The effect of cold therapy on the postoperative course of total hip and knee arthroplasty patients. Am J Orthop (Belle Mead NJ), 24(11), 847-852.

6. Abramson DI, Chu LS, Tuck S Jr, Lee SW, Richardson G, Levin M (1966) Effect of tissue temperatures and blood flow on motor nerve conduction velocity. JAMA 198(10), 1082-1088.

7. Matsen FA 3rd, Questad K, Matsen AL (1975) The effect of local cooling on postfracture swelling. A controlled study. Clin Orthop 109, 201-206.

8. Adie S, Naylor JM, Harris IA (2010) Cryotherapy after total knee arthroplasty: A systematic review and meta-analysis of randomized controlled trials. J Arthroplasty 25(5), 709-715.

9. Ivey M, Johnston RV, Uchida T (1994) Cryotherapy for postoperative pain relief following knee arthroplasty. J Arthroplasty 9(3), 285-290.

10. Kullenberg B, Ylipaa S, Soderlund K, Resch S (2006) Postoperative cryotherapy after total knee arthroplasty: A prospective study of 86 patients. J Arthroplasty 21(8), 1175-1179.

11. Iwakiri K, Ohta Y, Minoda $\mathrm{Y}$, Kobayashi A, Nakamura H (2019) Effect of periarticular morphine injection for total hip arthroplasty: A randomized, double-blind trial. Hip Int, 29(3), 245-252.

12. Nadler SB, Hidalgo JH, Bloch T (1962) Prediction of blood volume in normal human adults. Surgery 51(2), 224.

13. Stalman A, Berglund L, Dungnerc E, Arner P, Fellander-Tsai L (2011) Temperature-sensitive release of prostaglandin $E_{2}$ and diminished energy requirements in synovial tissue with postoperative cryotherapy. J Bone Joint Surg Am 93(21), 1961-1968.

14. Nicolaides AN, Breddin HK, Fareed J, Goldhaber S, Haas S, Hull R, Kalodiki E, Myers K, Samama M, Sasahara A (2001) Prevention of venous thromboembolism, International Consensus Statement. Guidelines compiled in accordance with the scientific evidence. Int Angiol 20(1), 1-37.

15. Busch CA, Whitehouse MR, Shore BJ, MacDonald SJ, McCalden RW, Bourne RB (2010) The efficacy of periarticular multimodal drug infiltration in total hip arthroplasty. Clin Orthop Relat Res 468(8), 2152-2159.

16. Melvin JS, Stryker LS, Sierra RJ (2015) Tranexamic acid in hip and knee arthroplasty. J Am Acad Orthop Surg 23(12), 732-740.

Cite this article as: Iwakiri K, Kobayashi A, Takeuchi Y, Kimura Y, Ohta Y \& Nakamura H (2019) Efficacy of continuous local cryotherapy following total hip arthroplasty. SICOT-J, 5, 13 